Received: 02.07.2021

Revised: 22.08 .2021

Accepted: 27.08 .2021

DOI: $10.17804 / 2410-9908.2021 .4 .34-49$

\title{
ANALYTICAL STUDY OF THE EKMAN ANGLE FOR THE BENARD-MARANGONI CONVECTIVE FLOW OF VISCOUS INCOMPRESSIBLE FLUID
}

\author{
A. V. Gorshkov ${ }^{\text {a) }}$ and E. Yu. Prosviryakov ${ }^{\text {b)* }}$ \\ Institute of Engineering Science, Ural Branch of the Russian Academy of Sciences, \\ 34 Komsomolskaya St., Ekaterinburg, 620049, Russian Federation \\ a) iD https://orcid.org/0000-0002-5741-3979 alex55gor@mail.ru; \\ b)iD https://orcid.org/0000-0002-2349-7801 《evgen_pros@mail.ru \\ *Corresponding author. E-mail: evgen_pros@mail.ru \\ Address for correspondence: 34 Komsomolskaya St., Ekaterinburg, 620049, Russian Federation \\ Tel.: +7 (343) 37535 76; fax: 3745330
}

The paper considers the convective flow of a viscous incompressible fluid over a rotating surface. It studies the angle between the fluid velocity vector in the upper layer and the temperature gradient vector on the free surface. For the study, an analytical solution to the Oberbeck-Boussinesq equations is constructed, which describes the stratified Ekman flow taking into account two components of the Coriolis force. The temperature gradient and the conditions of Marangoni thermocapillary convection are set at the upper (free) boundary, and the condition of fluid adhesion is set on the lower (solid) boundary. The representation of velocities in the form of linear functions of horizontal coordinates is used. It is shown that, when the flow depth tends to infinity, the angle between the upper layer fluid velocity vector and the temperature gradient vector tends to $\pi / 2$.

Keywords: analytical solution, thermocapillary convection, Ekman flow.

\section{References}

1. Pedlosky J. Geophysical fluid dynamics, Berlin, New York, Springer, 1987.

2. $\quad$ Gill A.E. Atmosphere-Ocean Dynamics, New York, Academic Press, 1982.

3. Ekman V.W. On the influence of the Earth's rotation on ocean-currents. Ark. Mat. Astron. Fys., 1905, vol. 2, No. 11, pp. 1-52.

4. Aristov S.N., Knyazev D.V., Polyanin A.D. Exact solutions of the Navier-Stokes Equations with the linear dependence of velocity components on two space variables. Theoretical Foundations of Chemical Engineering, 2009, vol. 43, No. 5, pp. 642-662. DOI: 10.1134/S0040579509050066.

5. Burmasheva N.V., Prosviryakov E.Yu. Exact solution of Navier-Stokes equations describing spatially inhomogeneous flows of a rotating fluid. Trudy Instituta Matematiki i Mekhaniki UrO RAN, 2020, vol. 26, No. 2, pp. 79-87. (In Russian).

6. Burmasheva N.V., Prosviryakov E.Yu. A class of exact solutions for two-dimensional equations of geophysical hydrodynamics with two Coriolis parameters. The Bulletin of Irkutsk State University. Series Mathematics, 2020, vol. 32, pp. 33-48. DOI: 10.26516/1997-7670.2020.32.33. (In Russian).

7. $\quad$ Felzenbaum A.I. Teoreticheskie osnovy i metody rascheta ustanovivshikhsya morskikh techeniy [Theoretical Foundations and Methods for Calculating Steady Sea Currents]. AN SSSR Publ., 1960, 127 p. (In Russian). 
8. Dolgansky F.V. Lektsii po geophizicheckoy gidrodinamike [Lectures on Geophysical Hidrodynamics]. Moscow, IVM RAN Publ, 2006, 378 p. (In Russian).

9. Korotaev G.K., Mikhaylova E.N., Shapiro N.B. Teoriya ekvatorialnykh protivotecheniy $v$ Mirovom okeane [Theory of Equatorial Countercurrents in the World Ocean]. Kiev, Nauk. Dumka Publ., 1986, 208 p. (In Russian).

10. Zyryanov V.N. Teoriya ustanovivshchikhsya okeanicheskikh techeniy [Theory of Steady Ocean Currents]. Leningrad, Gidrometeoizdat Publ., 1985. (In Russian).

11. Aristov S.N., Prosviryakov E.Yu. Aristov S.N., Prosviryakov E.Y. On laminar flows of planar free convection. Rus. J. Nonlin. Dyn., 2013, vol. 9, No. 4, pp. 651-657. DOI: 10.20537/nd1304004. (In Russian).

12. Aristov S.N., Shvarts K.G. Vikhrevye techeniya advektivnoy prirody vo vrashchayushchemsya sloe zhidkosti [Vortical Flows of Advective Nature in a Rotating Fluid Layer]. Perm, Perm. Gos. Univ. Publ., 2006, 154 p. (In Russian).

13. Aristov S.N., Shvarts K.G. Vikhrevye techeniya $v$ tonkikh sloyakh zhidkosti [Vortical Flows in Thin Fluid Layers]. Kirov, VyatGU Publ., 2011. (In Russian).

14. Aristov S.N., Shvarts K.G. Advective flow in a rotating liquid film. Journal of Applied Mechanics and Technical Physics, 2016, vol. 57, No. 1, pp. 188-194. DOI: 10.1134/S0021894416010211.

15. Ingel L.Kh., Aristov S.N. The class of exact solutions of nonlinear problems on thermal circulation associated with volumetric heat release in the atmosphere. Tr. In-ta Eksperim. Meteorol., 1996, No. 27 (162), pp. 142-157. (In Russian).

16. Gorshkov A.V., Prosviryakov E.Yu. Convective flow in the solid rotation of a viscous incompressible fluid. AIP Conference Proceedings, 2017, vol. 1915, 040020. DOI: 10.1063/1.5017368.

17. Gorshkov A.V., Prosviryakov E.Yu. Ekman Convective Layer Flow of a Viscous Incompressible Fluid. Izvestiya, Atmospheric and Oceanic Physics, 2018, vol. 54, No. 2, pp. 189-195. DOI: $10.1134 /$ S0001433818020081.

18. Gorshkov A.V. Prosviryakov E.Yu. Analytical Study of the Ekman Angle for the Isothermal Flow of a Viscous Incompressible Fluid in View of the Navier Boundary Condition. AIP Conference Proceedings, 2020, vol. 2315, 020018. DOI: 10.1063/5.0036889.

19. Constantin A., Johnson R.S. Atmospheric Ekman flows with variable eddy viscosity. Boundary-Layer Meteorology, 2019, vol. 170, pp. 395-414. DOI: 10.1007/s10546-018-0404-0.

20. Shrira V.I., Almelah R.B. Upper-ocean Ekman current dynamics: a new perspective. Journal of Fluid Mechanics, 2020, vol. 887, A24. DOI: 10.1017/jfm.2019.1059.

21. Fečkan M., Guan Y., O'Regan D., Wang J.R. Existence and uniqueness and first order approximation of solutions to atmospheric Ekman flows. Monatshefte für Mathematik, 2020, 193, pp. 623-636. DOI: 10.1007/s00605-020-01414-7.

22. Ortiz-Tarin J.L., Lee S., Flores O., Sarkar S. Global modes and large-scale structures in an Ekman boundary layer. Journal of Physics: Conference Series, 2020, vol. 1522, 012011. DOI: $10.1088 / 1742-6596 / 1522 / 1 / 012011$.

23. Prosviryakov E.Y. New class of exact solutions of Navier-Stokes equations with exponential dependence of velocity on two spatial coordinates. Theoretical Foundations of Chemical Engineering, 2019, vol. 53, No. 1, pp. 107-114. DOI: 10.1134/S0040579518060088.

24. Aristov S.N., Prosviryakov E.Y. A new class of exact solutions for three-dimensional thermal diffusion equations. Theoretical Foundations of Chemical Engineering, 2016, vol. 50, No. 3, pp. 286-293. DOI: 10.1134/S0040579516030027.

25. Burmasheva N.V., Prosviryakov E.Yu. Isothermal layered flows of a viscous incompressible fluid with spatial acceleration in the case of three Coriolis parameters. Diagnostics, Resource and 
Mechanics of materials and structures, 2020, iss. 3, pp. 29-46. DOI: 10.17804/24109908.2020.3.029-046. Available at: http://dream-journal.org/issues/2020-3/2020-3_291.html

26. Burmasheva N.V., Privalova V.V., Prosviryakov E.Yu. Layered Marangoni convection with the Navier slip condition. SADHANA-ACADEMY PROCEEDINGS IN ENGINEERING SCIENCES, 2021, vol. 46, iss. 1, No. 55. DOI: 10.1007/s12046-021-01585-5.

27. Ershkov Sergey V., Prosviryakov Evgeniy Yu., Burmasheva Natalya V., and Christianto Victor. Towards understanding the algorithms for solving the Navier-Stokes equations. Fluid Dynamics Research, 2021, vol. 53, No. 4, pp. 044501. DOI:10.1088/1873-7005/ac10f0. 
Подана в журнал: 02.07.2021

УДК 517.958

DOI: $10.17804 / 2410-9908.2021 .4 .34-49$

\title{
АНАЛИТИЧЕСКОЕ ИССЛЕДОВАНИЕ УГЛА ЭКМАНА ДЛЯ КОНВЕКТИВНОГО ТЕЧЕНИЯ БЕНАРА-МАРАНГОНИ ВЯЗКОЙ НЕСЖИМАЕМОЙ ЖИДКОСТИ
}

\author{
А. В. Горшков ${ }^{\text {a) }}$, Е. Ю. Просвиряков ${ }^{\text {б)* }}$ \\ Институт машиноведения УрО РАН, \\ ул. Комсомольская, 34, г. Екатеринбург, Российская Федерация \\ a) iD https://orcid.org/0000-0002-5741-3979 alex55gor@mail.ru; \\ б)(iD https://orcid.org/0000-0002-2349-7801 《 evgen_pros@mail.ru \\ *Ответственный автор. Электронная почта: evgen_pros@mail.ru \\ Адрес для переписки: ул. Комсомольская, 34, г. Екатеринбург, Российская Федерация \\ Тел.: +7 (343) 375-35-76; факс: 374-53-30
}

В работе рассмотрено конвективное течение вязкой несжимаемой жидкости по вращающейся поверхности. Исследован угол между вектором скорости жидкости в верхнем слое и вектором градиента температуры на свободной поверхности. Для исследования построено аналитическое решение уравнений Обербека-Буссинеска, описывающее слоистое течение Экмана с учетом двух компонент силы Кориолиса. На верхней (свободной) границе задан градиент температуры и условия термокапиллярной конвекции Марангони. На нижней (твердой) границе задано условие прилипания жидкости. Использована представление скоростей в виде линейных функций горизонтальных координат. Показано, что при стремлении глубины потока к бесконечности угол между вектором скорости жидкости в верхнем слое и вектором градиента температуры стремится к $\pi / 2$.

Ключевые слова: аналитическое решение, термокапиллярная конвекция, течение Экмана.

\section{1. Введение}

При вращении вязкой жидкости возникает угол между вектором касательных напряжений и вектором скорости течения в верхнем слое. Существование этого угла обусловлено действием силы Кориолиса, генерирующей движение изначально покоящейся жидкости. В научной литературе угол между векторами касательных напряжений и скорости называется углом Экмана $[1,2]$. Аналитическое исследование величины этого угла является важной задачей геофизической гидродинамики $[1,2]$. В работе Экмана [3] рассмотрен простейший случай изобарического течения океана бесконечной глубины при задании на свободной границе касательных напряжений, описывающих трение воздуха о морскую воду. Напомним, что движение Экмана является изобарическим только в неинерциальной (вращающейся) системе координат, а в инерциальной системе координат давление описывается квадрикой второго порядка, которая является круговым параболоидом [4-6].

При выполнении прогностических и диагностических расчетов течений Мирового океана используется традиционное приближение ускорения Кориолиса, характеризующееся одним параметром вектора угловой скорости (первый параметр Кориолиса). Строго говоря, такой подход позволяет описывать движение жидкости только в высоких широтах, где можно пренебречь вторым параметром Кориолиса [7-10]. Как известно, данное приближение также применяют и в средних широтах, но с дополнительными ограничениями на характерные масштабы скоростей и других гидродинамических полей [10]. Обобщения сдвигового 
течения Экмана и исследование его устойчивости опубликованы в статьях [4-6, 11-18] и монографиях [7-10].

В библиографических источниках [4-6, 11-18] отражены исследования краевых и начально-краевых задач геофизической гидродинамики для широкого класса граничных условий на обеих границах горизонтального вращающегося бесконечного слоя жидкости. В работе [18] исследуется угол Экмана для изотермического течения вязкой несжимаемой жидкости с учетом выполнения условия скольжения жидкости по твердой поверхности согласно закону Навье. Использование условия проскальзывания или условия идеального скольжения позволяет учесть неровность дна [19-22].

При исследовании конвективных крупномасштабных течений важную роль играет не только гравитационный механизм, но и термокапиллярный. Особенно важно изучать так называемый сложный механизм конвекции, который проявляется в суперпозиции различных факторов, генерирующих конвекцию. Классы точных решений для изотермических, конвективных и термодиффузионных течений в задачах гидродинамики вращающихся жидкостей анонсированы в научных статьях и библиографических обзорах $[4-6,14-17,23-27]$. При использовании семейств классов точных решений в данной работе исследуется сложная конвекция Бенара-Марангони вращающейся вязкой жидкости. Построено аналитическое выражение угла между вектором скорости и вектором градиента температуры на свободной поверхности. Градиент температуры на свободной поверхности создает касательные напряжения, вызывающие течение верхнего слоя жидкости (эффект Марангони). Исследования угла Экмана выполнены на основе нелинейных уравнений Навье-Стокса в приближении ОбербекаБуссинеска, описывающих слоистые течения жидкости с наклонной осью вращения.

\section{2. Постановка задачи}

Крупномасштабное сдвиговое конвективное течение в бесконечно протяженном горизонтальном слое вращающейся вязкой несжимаемой жидкости описывается системой уравнений Навье-Стокса в приближении Обербека-Буссинеска. Система уравнений, записанная в безразмерных переменных с учетом $V_{z}=0$, имеет вид $[5,6,12,14,16,17]$ :

$$
\begin{gathered}
\operatorname{Gr}\left(V_{x} \frac{\partial V_{x}}{\partial x}+V_{y} \frac{\partial V_{x}}{\partial y}\right)-\frac{\sin \varphi}{E k} V_{y}=-\mathrm{Gr} \frac{\partial P}{\partial x}+\Delta V_{x} ; \\
\operatorname{Gr}\left(V_{x} \frac{\partial V_{y}}{\partial x}+V_{y} \frac{\partial V_{y}}{\partial y}\right)+\frac{\sin \varphi}{E k} V_{x}=-\operatorname{Gr} \frac{\partial P}{\partial y}+\Delta V_{y} ; \\
\frac{\partial P}{\partial z}=\frac{2 \delta V_{x}}{\operatorname{Gr} \operatorname{Ek}} \cos \varphi+\frac{\delta}{\mathrm{Gr}} T ; \\
\operatorname{Ra}\left(V_{x} \frac{\partial T}{\partial x}+V_{y} \frac{\partial T}{\partial y}\right)=\Delta T ; \\
\frac{\partial V_{x}}{\partial x}+\frac{\partial V_{y}}{\partial y}=0 .
\end{gathered}
$$

Здесь $V_{x}$ и $V_{y}$ - безразмерные компоненты вектора скорости жидкости; $P$ - давление, нормированное на удвоенную удельную кинетическую энергию $\rho V^{2}$; безразмерные горизонтальные координаты $x$ и $y$ определяются характерным масштабом длины $L$, а поперечная координа- 
та $z$ - толщиной $h$ слоя жидкости; $\delta=h / L$ - отношение масштабов длины (относительная толщина потока); $T$ - безразмерная температура; $v$ - кинематическая вязкость жидкости; $\beta$ - коэффициент объемного расширения жидкости; $g$ - ускорение свободного падения; $\Omega$ - угловая скорость; $\varphi$ - угол наклона оси вращения к плоскости течения жидкости. Кроме того, для записи системы уравнений (1) использованы безразмерные комплексы: $\mathrm{Gr}=g \beta \vartheta L^{3} / v^{2}-$ число Грасгофа, где $\vartheta$ - разность максимальной и минимальной температур; $\operatorname{Ra}=\operatorname{Pr} \mathrm{Gr}-$ число Рэлея; $\operatorname{Pr}=v / \chi-$ число Прандтля; $\mathrm{Ek}=\mathrm{Ro} / \operatorname{Re}=v /\left(L^{2} \Omega\right)$ - число Экмана, где $\operatorname{Ro}=V /(L \Omega)$ - число Россби; $\operatorname{Re}=V L / \nu(V-$ характерный масштаб линейной скорости). Для удобства и краткости записи введен $\Delta=\frac{\partial^{2}}{\partial x^{2}}+\frac{\partial^{2}}{\partial y^{2}}+\frac{1}{\delta^{2}} \frac{\partial^{2}}{\partial z^{2}}-$ трехмерный оператор Лапласа.

Использование бесконечного горизонтального слоя для изучения течения вращающейся жидкости означает, что предложенная математическая модель (1) описывает крупномасштабные течения. В этом случае можно пренебречь кривизной планеты и рассматривать движение жидкости в области, принадлежащей касательному пространства тела, имеющего конечное значение кривизны.

Для системы (1) зададим следующие граничные условия. На нижней поверхности слоя жидкости, задаваемой уравнением $z=0$, температура определяется как линейная функция горизонтальных координат:

$$
\left.T\right|_{z=0}=A_{0}+x A_{1}+y A_{2}
$$

где $A_{0}$ - отсчетное значение фоновой температуры (можно принять равным нулю); $A_{1}$ и $A_{2}-$ постоянные. Также на нижней поверхности слоя жидкости задается условие прилипания

$$
\left.V_{x}\right|_{z=0}=0,\left.V_{y}\right|_{z=0}=0 .
$$

Верхняя свободная поверхность жидкости предполагается плоской и задается уравнением $z=1$ поскольку эта безразмерная координата получена делением на толщину слоя. На ней задана температура в виде, аналогичном (2):

$$
\left.T\right|_{z=1}=B_{0}+x B_{1}+y B_{2}
$$

где $B_{0}$ - значение фоновой температуры; $B_{1}$ и $B_{2}$ - постоянные. Давление на верхней границе считаем постоянным, не зависящим от продольных координат:

$$
\left.P\right|_{z=1}=S
$$

где $S$ - постоянная, которую можно принять за отсчетное значение и положить равной нулю.

На свободной поверхности действует термокапиллярный эффект Марангони-Гиббса. Граничное условие Марангони в безразмерных переменных примет вид:

$$
\left.\frac{\partial V_{x}}{\partial z}\right|_{z=1}=-\operatorname{Mg} \frac{\partial T}{\partial x},\left.\frac{\partial V_{y}}{\partial z}\right|_{z=1}=-\operatorname{Mg} \frac{\partial T}{\partial y}
$$

где $\mathrm{Mg}$ - безразмерное число Марангони. 
Анализ системы (1) показывает, что ее разрешимость возможна в простейшем полиномиальном классе решений, принадлежащем семейству Линя-Сидорова-Аристова [11-15, 24]:

$$
\begin{gathered}
V_{x}=U(z) ; V_{y}=V(z) ; \\
P=P_{0}(z)+x P_{1}(z)+y P_{2}(z) ; \\
T=T_{0}(z)+x T_{1}(z)+y T_{2}(z) .
\end{gathered}
$$

После подстановки вида решения (7) в систему уравнений (1) получим разрешающую систему обыкновенных дифференциальных уравнений для функций $U, V, T_{0}, T_{1}, T_{2}, P_{0}, P_{1}, P_{2}$ :

$$
\begin{gathered}
T_{1}^{\prime \prime}=0 ; T_{2}^{\prime \prime}=0 ; \\
P_{1}^{\prime}=-\frac{\delta}{G r} T_{1} ; P_{2}^{\prime}=-\frac{\delta}{G r} T_{2} ; \\
U^{\prime \prime}+\frac{\delta^{2} \sin \varphi}{\mathrm{Ek}} V=\delta^{2} \mathrm{Gr} P_{1} ; V^{\prime \prime}-\frac{\delta^{2} \sin \varphi}{\mathrm{Ek}} U=\delta^{2} \mathrm{Gr} P_{2} ; \\
T_{0}^{\prime \prime}=R a \delta^{2}\left(T_{1} U+T_{2} V\right) ; \\
P_{0 T}{ }^{\prime}=-\frac{\delta}{G r} T_{0} \cos \varphi ; P_{0 U}^{\prime}=-\frac{\delta}{G r} \frac{U}{E k} \cos \varphi ; \\
P_{0}=P_{0 T}+P_{0 U} .
\end{gathered}
$$

Здесь штрих обозначает дифференцирование по переменной $z$. Фоновое давление $P_{0}$ для удобства разделено на два слагаемых. Слагаемое $P_{0 U}$ определяется компонентой скорости $U$ из системы уравнений (8) и (9), а слагаемое $P_{0 T}-$ фоновой температурой $T_{0}$.

Система уравнений (8)-(10) может быть последовательно проинтегрирована в том порядке, в котором выписаны уравнения.

При использовании данного класса точного решения (7) для системы (1) второй параметр Кориолиса содержится только в уравнении для определения давления $P_{0}(10)$, а поле скоростей, определяемое уравнениями (9), является обобщением классического решения Экмана [6-11]. Из уравнения (10) следует, что при учете двух параметров Кориолиса слоистое течение жидкости не может быть изобарическим во вращающейся системе координат.

Граничные условия (2)-(6), записанные для системы (1), в соответствии с классом решений (7) преобразуются к следующим граничным условиям системы (8)-(10): на нижней границе заданы компоненты вектора градиента граничной температуры и отсчетное значение фоновой температуры, которое можно положить равным нулю. Заданы также условия прилипания жидкости к твердой поверхности:

$$
\begin{gathered}
T_{1}(0)=A_{1} ; T_{2}(0)=A_{2} ; T_{0}(0)=A_{0} ; \\
U(0)=0 ; V(0)=0 .
\end{gathered}
$$

На верхней свободной поверхности заданы компоненты вектора градиента температуры, компоненты вектора градиента давления и условия Марангони:

$$
T_{1}(1)=B_{1} ; T_{2}(1)=B_{2} ; T_{0}(1)=B_{0}
$$




$$
\begin{gathered}
U^{\prime}(1)=-\delta M g T_{1}(1) ; V^{\prime}(1)=-\delta M g T_{2}(1) \\
P_{1}(1)=0 ; P_{2}(1)=0 ; P_{0}(1)=0 .
\end{gathered}
$$

Граничные условия (11) и (12) позволяют определить постоянные интегрирования общего решения системы дифференциальных уравнений (8)-(10).

\section{3. Течение Экмана, характеризующееся двумя параметрами Кориолиса}

При заданных граничных условиях (11) и (12) получились следующие точные полиномиальные решения системы уравнений (8), определяющие градиенты давления и температуры:

$$
\begin{gathered}
T_{1}=A_{1}+\left(B_{1}-A_{1}\right) z ; \\
T_{2}=A_{2}+\left(B_{2}-A_{2}\right) z ; \\
P_{1}=-\frac{\left(B_{1}\left(1-z^{2}\right)+A_{1}(z-1)^{2}\right) \delta}{2 \mathrm{Gr}} ; \\
P_{2}=-\frac{\left(B_{2}\left(1-z^{2}\right)+A_{2}(z-1)^{2}\right) \delta}{2 \mathrm{Gr}} .
\end{gathered}
$$

Так как для дальнейших исследований вектор градиента температуры на твердой поверхности не существенен, то будем без ограничения потери общности для простоты считать его равным нулю: $A_{1}=0 ; A_{2}=0$.

Система уравнений (9) является линейной неоднородной системой дифференциальных уравнений четвертого порядка. Уравнение собственных чисел соответствующей линейной однородной системы - алгебраическое уравнение 4-го порядка:

$$
\lambda^{4}+4\left(\frac{\sin \varphi}{2 \mathrm{Ek}}\right)^{2}=0 .
$$

Собственные числа системы уравнений (9), являющиеся корнями уравнения (13), равны:

$$
\begin{gathered}
\lambda_{1}=k(1+i) ; \lambda_{2}=k(1-i) ; \\
\lambda_{3}=k(-1+i) ; \lambda_{4}=k(-1-i),
\end{gathered}
$$

где для краткости обозначено $k=\sqrt{\frac{|\sin \varphi|}{2 \mathrm{Ek}}} \cdot$ Модуль $|\sin \varphi|$ взят с учетом того, что в южном полушарии для географической широты справедливо неравенство $\varphi<0$, а в уравнение (13) $\sin \varphi$ входит с квадратом.

Общее решение системы неоднородных уравнений (9) имеет вид:

$$
\begin{gathered}
U=\left(c_{1} \exp (k \delta z)+c_{3} \exp (-k \delta z)\right) \cos (k \delta z)+ \\
+\left(c_{2} \exp (k \delta z)+c_{4} \exp (-k \delta z)\right) \sin (k \delta z)+\frac{B_{1}-B_{2} z^{2} \delta^{2} k^{2}}{4 k^{4} \delta}
\end{gathered}
$$




$$
\begin{gathered}
V=\left(c_{4} \exp (-k z \delta)-c_{2} \exp (k \delta z)\right) \cos (k \delta z)+ \\
+\left(c_{1} \exp (k \delta z)-c_{3} \exp (-k \delta z)\right) \sin (k \delta z)+\frac{B_{2}+B_{1} z^{2} \delta^{2} k^{2}}{4 k^{4} \delta},
\end{gathered}
$$

где $c_{1}, c_{2}, c_{3}, c_{4}$ - произвольные постоянные интегрирования, определяемые из граничных условий (11) и (12). Полное частное решение системы (9), удовлетворяющее заданным граничным условиям (11) и (12), слишком громоздко и здесь не приводится. По этой же причине не приводятся выражения фоновой температуры и давления.

При постоянной, независящей от горизонтальных координат температуре на твердой поверхности решение для скоростей можно записать в виде:

$$
\begin{aligned}
& U=B_{1}\left(\frac{1}{4 k^{4} \delta}+f_{1}(z)+\operatorname{Mg} \varphi_{1}(z)\right)-B_{2}\left(\frac{z^{2} \delta}{4 k^{2}}+\operatorname{Mg} \varphi_{2}(z)-f_{2}(z)\right) \\
& V=B_{1}\left(\operatorname{Mg} \varphi_{2}(z)+\frac{z^{2} \delta}{4 k^{2}}-f_{2}(z)\right)+B_{2}\left(\operatorname{Mg} \varphi_{1}(z)+\frac{1}{4 k^{4} \delta}+f_{1}(z)\right) .
\end{aligned}
$$

Здесь функции $f_{1}(z) ; f_{2}(z) ; \varphi_{1}(z) ; \varphi_{2}(z)$ имеют следующий вид:

$$
\begin{aligned}
& \varphi_{2}(z)=[\cosh (k \delta(z+1)) \sin (k \delta(z-1))+\cosh (k \delta(z-1)) \sin (k \delta(z+1))+ \\
& +\sinh (k \delta(1+z)) \cos (k \delta(z-1))-\sinh (k \delta(z-1)) \cos (k \delta(z-1))] \times \\
& \times[2 k \delta(\cosh (2 k \delta)+\cos (2 k \delta))]^{-1} ; \\
& \varphi_{1}(z)=[\sinh (k \delta(z+1)) \cos (k \delta(z-1))+\sinh (k \delta(z-1)) \cos (k \delta(z+1))+ \\
& +\cosh (k \delta(z-1)) \sin (k \delta(z-1))+\cosh (k \delta(z-1)) \sin (k \delta(z+1))] \times \\
& \times[2 k \delta(\cosh (2 k \delta)+\cos (2 k \delta))]^{-1} ; \\
& f_{1}(z)=[-\cosh (k \delta z) \cos (k \delta(z-1))-k \delta \sinh (k \delta(z+1)) \cos (k \delta(z-1))- \\
& -\cosh (k \delta(z-2)) \cos (k \delta z)-k \delta \sinh (k \delta(z-1)) \cos (k \delta(z+1))+ \\
& +k \delta \cosh (k \delta(z+1)) \sin (k \delta(z-1))+k \delta \cosh (k \delta(z-1)) \sin (k \delta(z+1))] \times \\
& \times\left[4 k^{4} \delta(\cosh (2 k \delta)+\cos (2 k \delta))\right] \\
& f_{2}(z)=\sinh (k \delta z) \sin (k \delta(z-2))-\sinh (k \delta(z-2)) \sin (k \delta z)- \\
& -k \delta \sinh (k \delta(1+z)) \cos (k \delta(z-1))+k \delta \sinh (k \delta(z-1)) \cos (k \delta(1+z))- \\
& -k \delta \cosh (k \delta(1+z)) \sin (k \delta(z-1))-k \delta \cosh (k \delta(z-1)) \sin (k \delta(1+z))] \times \\
& \times\left[4 k^{4} \delta(\cosh (2 \delta k)+\cos (2 \delta k))\right]^{-1} \cdot
\end{aligned}
$$

Поле скоростей (15) принадлежит классу тригонометрических квазиполиномов и далее будет исследовано. 


\section{4. Асимптотическое приближение скоростей}

Рассмотрим конвективное течение при больших значениях относительной толщины потока $\delta$. Будем считать, что произведение $k \delta$ достаточно велико и можно пренебречь слагаемыми, содержащими в качестве множителей экспоненты, показатель которых при всех значениях $z$ из отрезка $(0,1)$ меньше нуля. Приведенное условие позволяет рассматривать не только течения жидкости в тонком слое (малые значения относительной толщины $\delta$ ), но и очень медленные вращательные движения жидкости при толщинах, у которых значение $\delta$ больше единицы. Отметим, что при больших значениях параметра $\delta$ реализация условий, полученных ранее, возможна при медленном вращении жидкости (угловая скорость должна быть меньше, чем у планеты Земля).

Разделим числители и знаменатели выражений функций (15) на $\exp (4 k \delta)$ и оставим слагаемые, содержащие в качестве множителей экспоненты, показатель которых хотя бы при одном значении z из отрезка $(0,1)$ обращается в ноль: $\exp (-k \delta z) ; \exp (k \delta(z-1))$. Функции $f_{1} ; f_{2} ; \varphi_{1} ; \varphi_{2}$ принимают вид:

$$
\begin{gathered}
\varphi_{1}=\exp (k \delta(z-1)) \frac{\sqrt{2} \sin (k \delta(z-1)+\pi / 4)}{2 k \delta} ; \\
\varphi_{2}=\exp (k \delta(z-1)) \frac{\sqrt{2} \sin (k \delta(z-1)-\pi / 4)}{2 k \delta} ; \\
f_{1}=\frac{-\sqrt{2} \exp (k \delta(z-1)) \sin (k \delta(z-1)+\pi / 4)}{4 k^{3}}-\exp (-k \delta z) \frac{\cos (k \delta z)}{4 k^{4} \delta} ; \\
f_{2}=\frac{\sqrt{2} \exp (k \delta(z-1)) \sin (\pi / 4+k \delta(z-1))}{4 k^{3}}+\exp (-k \delta z) \frac{\sin (k \delta z)}{4 k^{4} \delta} .
\end{gathered}
$$

Из полученных выражений следует, что при больших значениях параметра $k \delta$ (для геофизической гидродинамики $\delta<1$ ) вторые слагаемые функций $f_{1} f_{2}$ экспоненциально затухают при стремлении координаты $z$ к единице, т. е.

$$
\begin{aligned}
& \lim _{\substack{z \rightarrow 1 \\
k \delta \rightarrow \infty}}\left(\frac{-\sqrt{2} \exp (k \delta(z-1)) \sin (k \delta(z-1)+\pi / 4)}{4 k^{3}}-\exp (-k \delta z) \frac{\cos (k \delta z)}{4 k^{4} \delta}\right)=0 \\
& \lim _{\substack{z \rightarrow 1 \\
k \delta \rightarrow \infty}}\left(\frac{\sqrt{2} \exp (k \delta(z-1)) \sin (\pi / 4+k \delta(z-1))}{4 k^{3}}+\exp (-k \delta z) \frac{\sin (k \delta z)}{4 k^{4} \delta}\right)=0
\end{aligned}
$$

Иными словами, на свободной поверхности функции $f_{1}$ и $f_{2}$ практически не вносят вклада в описание течения в приповерхностном слое. Отметим, первые слагаемые функций $f_{1}{ }_{\text {и }} f_{2}$, как и функции $\varphi_{1}$ и $\varphi_{2}$, на свободной поверхности ограничены, что говорит о их доминирующем вкладе во вращающийся поток жидкости.

На рис. 1 и 2 представлено наличие спиральных противотечений во вращающейся жидкости. Влияние гравитационной и термокапиллярной конвекции приводит к наличию 
двух нулевых точек в потоке жидкости, что отличает от классического точного решения Экмана [3].

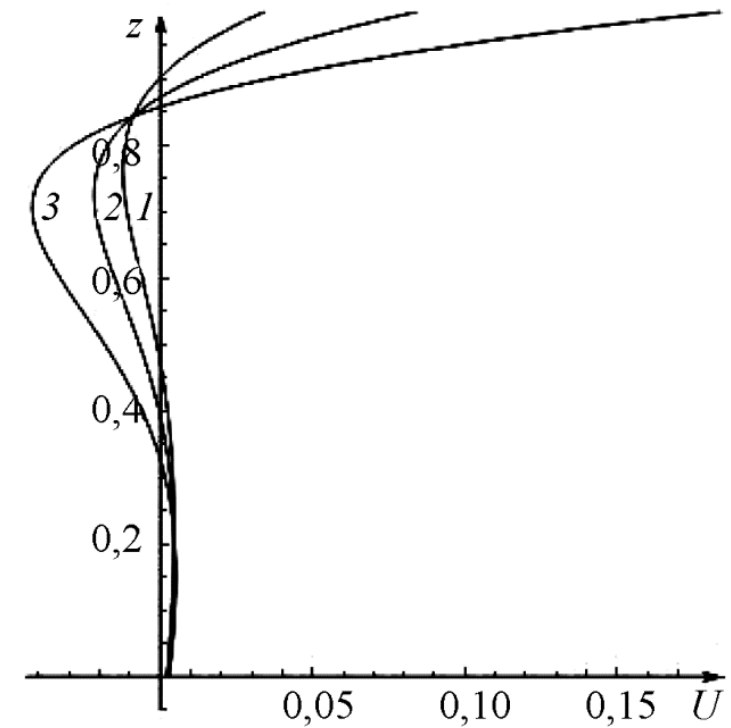

Рис. 1. Профили компоненты скорости $U$. Кривые построены при использовании следующих значений параметров:

$$
\begin{gathered}
1-M g=0,05 ; B_{1}=10 ; B_{2}=0 ; k \delta=5 ; \\
2-M g=0,1 ; B_{1}=10 ; B_{2}=0 ; k \delta=5 ; \\
3-M g=0,2 ; B_{1}=10 ; B_{2}=0 ; k \delta=5
\end{gathered}
$$

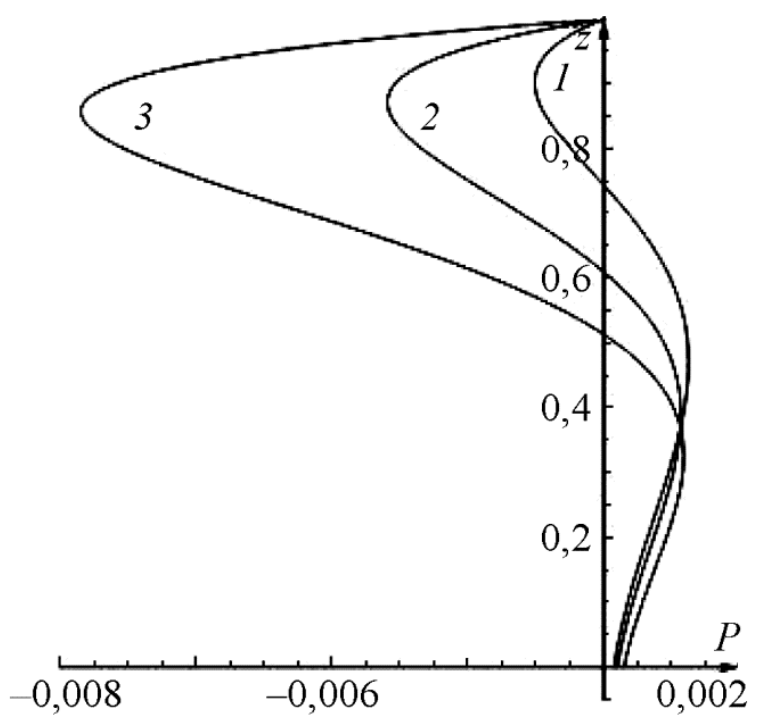

Рис. 3. Профиль слагаемого фонового давления $P_{0 U}$. Кривые построены при использовании следующих значений параметров:

$$
\begin{gathered}
1-M g=0,05 ; B_{1}=10 ; B_{2}=0 ; k \delta=5 ; \\
2-M g=0,1 ; B_{1}=10 ; B_{2}=0 ; k \delta=5 ; \\
3-M g=0,2 ; B_{1}=10 ; B_{2}=0 ; k \delta=5
\end{gathered}
$$

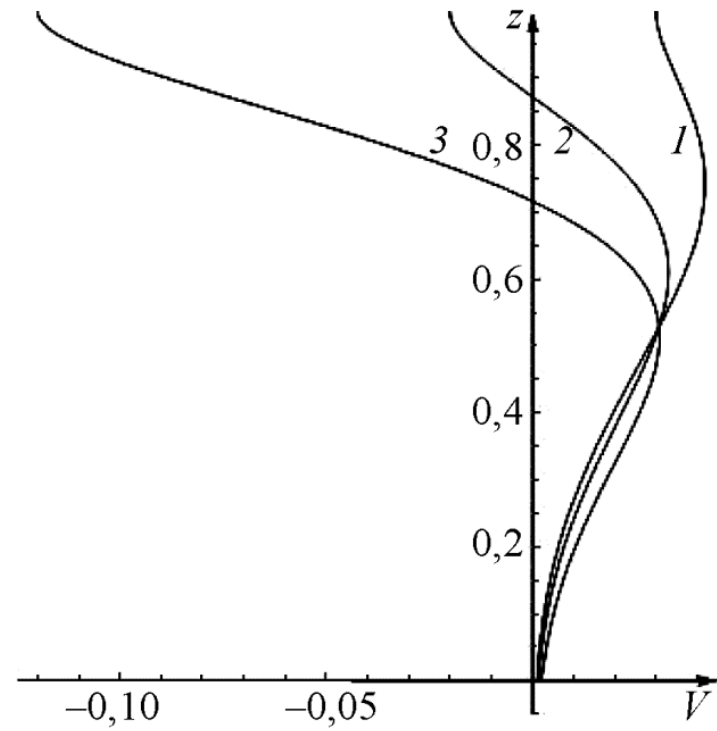

Рис. 2. Профили компоненты скорости $V$ Кривые построены при использовании следующих значений параметров:

$1-M g=0,05 ; B_{1}=10 ; B_{2}=0 ; k \delta=5$;

$2-M g=0,1 ; B_{1}=10 ; B_{2}=0 ; k \delta=5$;

$3-M g=0,2 ; B_{1}=10 ; B_{2}=0 ; k \delta=5$

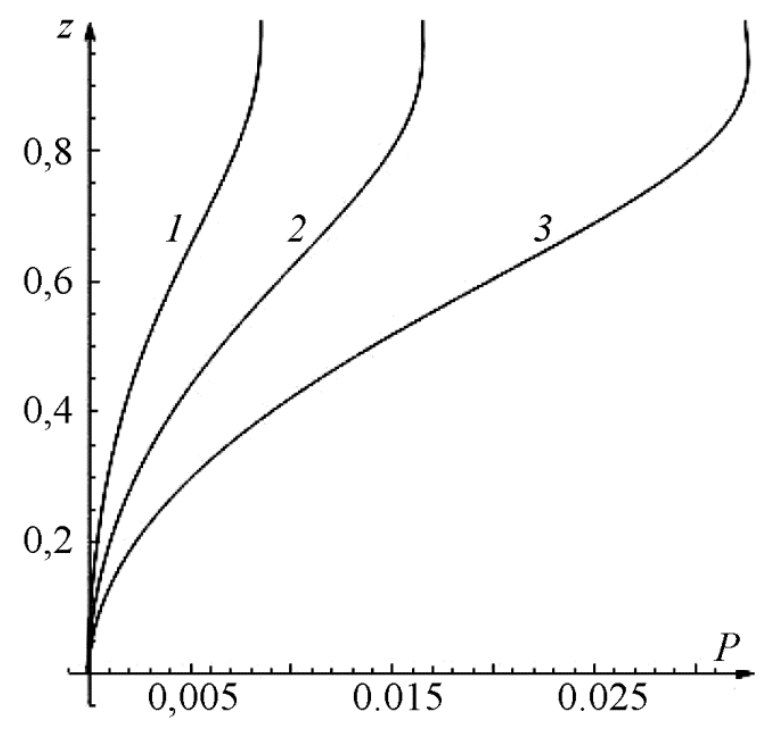

Рис. 4. Профиль слагаемого фонового давления $P_{0 T}$ Кривые построены при использовании следующих значений параметров:

$$
\begin{gathered}
1-M g=0,05 ; B_{1}=10 ; B_{2}=0 ; k \delta=5 ; \\
2-M g=0,1 ; B_{1}=10 ; B_{2}=0 ; k \delta=5 ; \\
3-M g=0,2 ; B_{1}=10 ; B_{2}=0 ; k \delta=5
\end{gathered}
$$




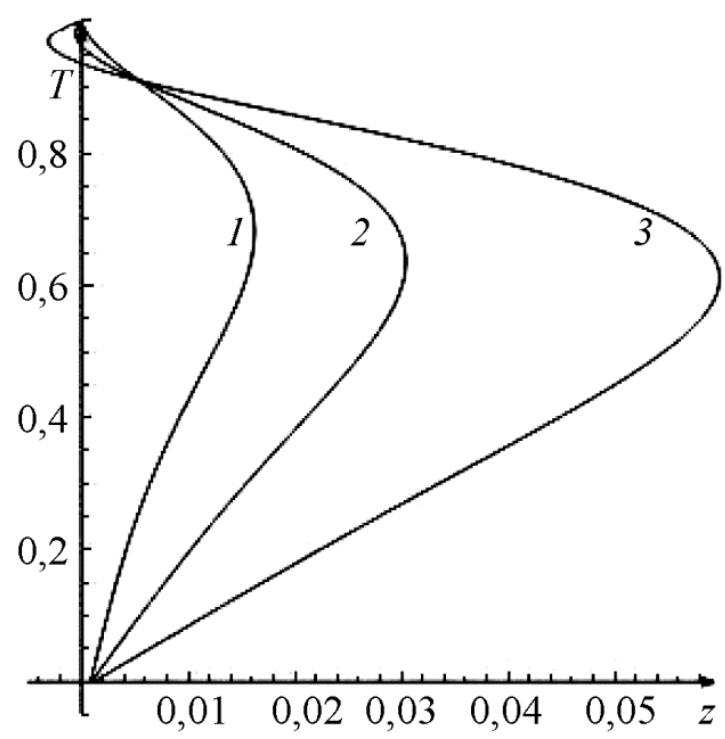

Рис. 5. Профили фоновой температуры $T_{0}$. Кривые построены при использовании следующих значений параметров: $1-M g=0,05 ; B_{1}=10 ; B_{2}=0 ; k \delta=5 ; 2-M g=0,1$;

$$
B_{1}=10 ; B_{2}=0 ; k \delta=5 ; 3-M g=0,2 ; B_{1}=10 ; B_{2}=0 ; k \delta=5
$$

Существование застойных точек приводит к стратификации полей температуры и давления (рис. 3-5). Расслоение полей температуры и давления свидетельствует о возникновении термоклина и пикноклина в тонком слое жидкости. Регистрация пикноклина посредством анализа гидродинамических полей при постоянной фоновой плотности обусловлена выбором приближения Буссинеска и пренебрежением сжимаемостью жидкости из-за температурного вклада для зависимости плотности от структурных параметров жидкости.

\section{5. Определение угла Экмана}

Исследуем угол между вектором скорости потока жидкости в верхнем слое и осью абсцисс $O x$. Тангенс угла $\gamma$ между вектором скорости и осью $O x$ при конечной глубине имеет вид:

$$
\operatorname{tg} \gamma(z)=\frac{B_{1}\left(M g \varphi_{2}(z)+\frac{z^{2} \delta}{4 k^{2}}-f_{2}(z)\right)+B_{2}\left(M g \varphi_{1}(z)+\frac{1}{4 k^{4} \delta}+f_{1}(z)\right)}{B_{1}\left(M g \varphi_{1}(z)+\frac{1}{4 k^{4} \delta}+f_{1}(z)\right)-B_{2}\left(M g \varphi_{2}(z)+\frac{z^{2} \delta}{4 k^{2}}-f_{2}(z)\right)} .
$$

Введем угол $\gamma_{\mu}$ :

$$
\operatorname{tg} \gamma_{\mu}=\frac{\frac{z^{2} \delta}{4 k^{2}}+M g \varphi_{2}(z)-f_{2}(z)}{\frac{1}{4 k^{4} \delta}+M g \varphi_{1}(z)+f_{1}(z)}
$$


Угол $\gamma_{B}$ определим из условия $\operatorname{tg} \gamma_{B}=\frac{B_{2}}{B_{1}}$. Проведя элементарные преобразования выражения $\operatorname{tg} \gamma(16)$, получим:

$$
\operatorname{tg} \gamma=\frac{\frac{M g \varphi_{2}(z)+\frac{z^{2} \delta}{4 k^{2}}-f_{2}(z)}{M g \varphi_{1}(z)+\frac{1}{4 k^{4} \delta}+f_{1}(z)}+\frac{B_{2}}{B_{1}}}{1-\frac{B_{2}}{B_{1}} \frac{M g \varphi_{2}(z)+\frac{z^{2} \delta}{4 k^{2}}-f_{2}(z)}{M g \varphi_{1}(z)+\frac{1}{4 k^{4} \delta}+f_{1}(z)}}=\operatorname{tg}\left(\gamma_{B}+\gamma_{\mu}\right)
$$

Таким образом, угол $\gamma$ складывается из двух слагаемых - угла между вектором граничного градиента температуры $\left(B_{1}\right.$ ѐ $\left.B_{2}\right)$, осью $O x$ и угла, зависящего от относительной толщины слоя жидкости $\delta$, глубины $z$, скорости вращения, определяемой параметром $k$ и числом Марангони $\mathrm{Mg}$.

Для определения угла между вектором скорости потока и осью $O x$ при большой глубине вычислим предел выражения:

$$
\begin{gathered}
\lim _{\delta \rightarrow \infty} \frac{B_{1}\left(\operatorname{Mg} \varphi_{2}(z)+\frac{z^{2} \delta}{4 k^{2}}-f_{2}(z)\right)+B_{2}\left(M g \varphi_{1}(z)+\frac{1}{4 k^{4} \delta}+f_{1}(z)\right)}{B_{1}\left(\operatorname{Mg} \varphi_{1}(z)+\frac{1}{4 k^{4} \delta}+f_{1}(z)\right)-B_{2}\left(\operatorname{Mg} \varphi_{2}(z)+\frac{z^{2} \delta}{4 k^{2}}-f_{2}(z)\right)}= \\
=\frac{B_{1}}{-B_{2}}=-\operatorname{ctg} \gamma_{B}=\operatorname{tg}\left(\gamma_{B}+\pi / 2\right) .
\end{gathered}
$$

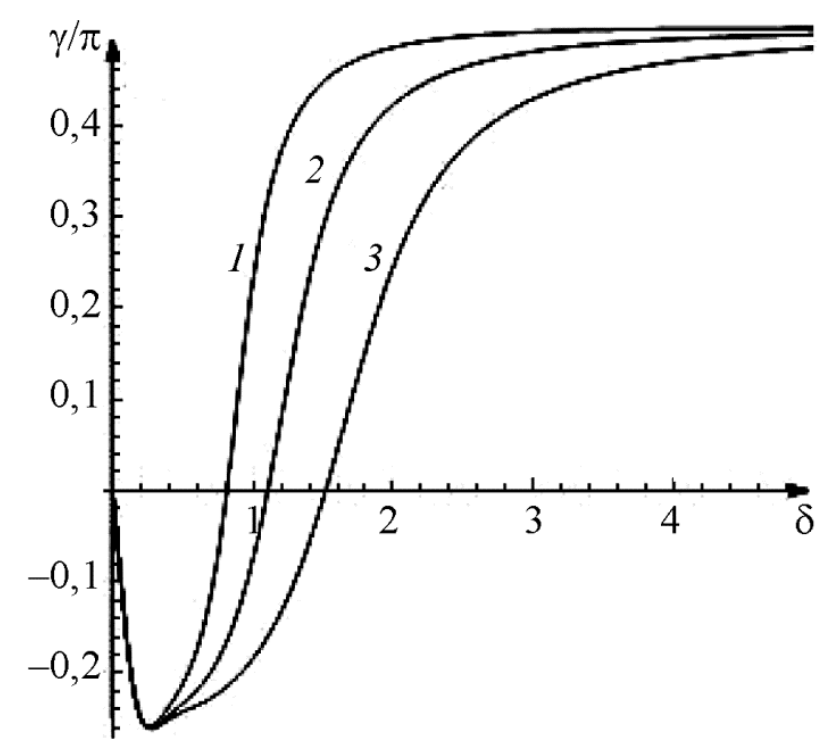

Рис. 6. График изменения угла $\gamma_{\mu}$ в верхнем слое с ростом относительной глубины $\delta$. Кривые построены при использовании следующих значений параметров: $1-M g=0,05$; 


$$
\begin{gathered}
B_{1}=10 ; B_{2}=0 ; k \delta=5 ; 2-M g=0,1 ; B_{1}=10 ; B_{2}=0 ; k \delta=5 ; \\
3-M g=0,2 ; B_{1}=10 ; B_{2}=0 ; k \delta=5
\end{gathered}
$$

Следовательно, угол между вектором скорости жидкости и вектором градиента температуры стремится к $\pi / 2$ при стремлении глубины к бесконечности (рис. 6). Наиболее интересен для задач геофизической гидродинамики противоположный предельный переход, когда относительная толщина $\delta$ близка нулю. В этом случае, как показывает анализ формулы (16), угол Экмана всегда тупой и зависит от граничных условий и параметров жидкости.

\section{5. Заключение}

В работе получено аналитическое решение уравнений Навье-Стокса, описывающее конвекцию Бенара-Марангони вязкой несжимаемой жидкости. Построено аналитическое выражение угла Экмана конвективного течения несжимаемой жидкости. Проведено аналитическое исследование угла Экмана. Угол представлен в виде суммы двух углов - угла между вектором градиента температуры и осью $O x$ и угла, определяемого параметрами потока. Показано, что при большой глубине вектор скорости потока на свободной поверхности поворачивается на $\gamma_{\mu}=\pi / 2$ относительно вектора градиента температуры. Предельное значение угла поворота вектора скорости $\gamma_{\mu}$ не зависит от коэффициента Марангони.

\section{Литература}

1. Pedlosky J. Geophysical fluid dynamics. - Berlin, New York : Springer, 1987.

2. Gill A. E. Atmosphere-Ocean Dynamics. - New York : Academic Press, 1982.

3. Ekman V. W. On the influence of the Earth's rotation on ocean-currents // Ark. Mat. Astron. Fys. - 1905. - Vol. 2, No. 11. - P. 1-52.

4. Aristov S. N., Knyazev D. V., Polyanin A. D. Exact solutions of the Navier-Stokes Equations with the linear dependence of velocity components on two space variables // Theoretical Foundations of Chemical Engineering. - 2009. - Vol. 43, No. 5. - P. 642-662. DOI: $10.1134 / \mathrm{S} 0040579509050066$.

5. Бурмашева Н. В., Просвиряков Е. Ю. Точное решение уравнений Навье-Стокса, описывающее пространственно неоднородные течения вращающейся жидкости // Труды Института математики и механики УрО РАН. - 2020. - Т. 26, вып. 2. - С. 79-87.

6. Бурмашева Н. В., Просвиряков Е. Ю. Класс точных решений для двумерных уравнений геофизической гидродинамики с двумя параметрами Кориолиса // Известия Иркутского государственного университета. Серия Математика. - 2020. - Т. 32. - С. 33-48. DOI: $10.26516 / 1997-7670.2020 .32 .33$.

7. Фельзенбаум А. И. Теоретические основы и методы расчета установившихся морских течений. - М. : Изд-во Акад. наук СССР, 1960. - 127 с.

8. Должанский Ф. В. Лекции по геофизической гидродинамике. - М. : ИВМ РАН, 2006. $378 \mathrm{c}$.

9. Коротаев Г. К., Михайлова Э. Н., Шапиро Н. Б. Теория экваториальных противотечений в Мировом океане. - Киев : Наук. думка, 1986. - 208 с. 
10. Зырянов В. Н. Теория установившихся океанических течений. - Л. : Гидрометеоиздат, 1985. - 248 c.

11. Аристов С. Н., Просвиряков Е. Ю. О слоистых течениях плоской свободной конвекции // Нелинейная динамика. - 2013. - Т. 9, № 3. - С. 3-9.

12. Аристов С. Н., Шварц К. Г. Вихревые течения адвективной природы во вращающемся слое жидкости. - Пермь : ПГУ, 2006. - 155 с.

13. Аристов С. Н., Шварц К. Г. Вихревые течения в тонких слоях жидкости. - Киров : ВятГУ, 2011. - 207 с.

14. Aristov S. N., Shvarts K. G. Advective flow in a rotating liquid film // Journal of Applied Mechanics and Technical Physics. - 2016. - Vol. 57, No. 1. - P. 188-194. DOI: $10.1134 / \mathrm{S} 0021894416010211$.

15. Ингель Л. Х., Аристов С. Н. Класс точных решений нелинейных задач о термических циркуляциях, связанных с объемным тепловыделением в атмосфере // Тр. Ин-та экспериментальной метеорологии. - 1996. - Вып. 27 (162). - С. 142-157.

16. Gorshkov A. V., Prosviryakov E. Yu. Convective flow in the solid rotation of a viscous incompressible fluid // AIP Conference Proceedings. - 2017. - 1915. - 040020. DOI: $10.1063 / 1.5017368$.

17. Gorshkov A. V., Prosviryakov E. Yu. Ekman Convective Layer Flow of a Viscous Incompressible Fluid // Izvestiya, Atmospheric and Oceanic Physics. - 2018. - Vol. 54, No. 2. - P. 189-195. DOI: $10.1134 /$ S0001433818020081.

18. Gorshkov A. V. Prosviryakov E. Yu. Analytical Study of the Ekman Angle for the Isothermal Flow of a Viscous Incompressible Fluid in View of the Navier Boundary Condition // AIP Conference Proceedings. - 2020. - Vol. 2315. - 020018. - DOI: 10.1063/5.0036889.

19. Constantin A., Johnson R. S. Atmospheric Ekman flows with variable eddy viscosity // Boundary-Layer Meteorology. - 2019. - Vol. 170. - P. 395-414. - DOI: 10.1007/s10546-0180404-0.

20. Shrira V. I., Almelah R. B. Upper-ocean Ekman current dynamics: a new perspective // Journal of Fluid Mechanics. - 2020. - Vol. 887. - A 24. - DOI: 10.1017/jfm.2019.1059.

21. Existence and uniqueness and first order approximation of solutions to atmospheric Ekman flows / M. Fečkan, Y. Guan, D. O’Regan, J. R. Wang // Monatshefte für Mathematik. - 2020. 193. - P. 623-636. - DOI: 10.1007/s00605-020-01414-7.

22. Global modes and large-scale structures in an Ekman boundary layer / J. L. Ortiz-Tarin, S. Lee, O. Flores, S. Sarkar // Journal of Physics: Conference Series. - 2020. - Vol. 1522. P. 012011. - DOI: 10.1088/1742-6596/1522/1/012011.

23. Prosviryakov E. Y. New class of exact solutions of Navier-Stokes equations with exponential dependence of velocity on two spatial coordinates // Theoretical Foundations of Chemical Engineering. - 2019. - Vol. 53, No. 1. -P. 107-114. - DOI: 10.1134/S0040579518060088.

24. Aristov S. N., Prosviryakov E. Y. A new class of exact solutions for three-dimensional thermal diffusion equations // Theoretical Foundations of Chemical Engineering. - 2016. - Vol. 50, No. 3. - P. 286-293. - DOI: 10.1134/S0040579516030027.

25. Burmasheva N. V., Prosviryakov E. Yu. Isothermal layered flows of a viscous incompressible fluid with spatial acceleration in the case of three Coriolis parameters // Diagnostics, Resource and Mechanics of materials and structures. - 2020. - Iss. 3. - P. 29-46. DOI: 10.17804/2410-9908.2020.3.029-046. - URL: http://dream-journal.org/issues/20203/2020-3_291.html 
26. Burmasheva N. V., Privalova V. V., Prosviryakov E. Yu. Layered Marangoni convection with the Navier slip condition // SADHANA-ACADEMY PROCEEDINGS IN ENGINEERING SCIENCES. - 2021. - Vol. 46, iss. 1, No. 55. - DOI: 10.1007/s12046-021-01585-5.

27. Towards understanding the algorithms for solving the Navier-Stokes equations / Sergey V. Ershkov, Evgeniy Yu. Prosviryakov, Natalya V. Burmasheva, and Victor Christianto // Fluid Dynamics Research. - 2021. - Vol. 53, No. 4. - P. 044501. - DOI:10.1088/18737005/ac10f0. 